\title{
Influence of different post-thaw culture time on the clinical outcomes of different quality embryos
}

\author{
Hui Wang ${ }^{A-D, F}$, Zhanhui $O u^{A-D, F}$, Zhiheng Chen ${ }^{\mathrm{E}, F}$, Li Yang ${ }^{A-C, F}$, Ling Sun ${ }^{\mathrm{E}, \mathrm{F}}$ \\ Center of Reproductive Medicine, Guangzhou Women and Children's Medical Center, Guangzhou Medical University, China \\ A - research concept and design; $B$ - collection and/or assembly of data; $C$ - data analysis and interpretation; \\ $D$ - writing the article; $E$ - critical revision of the article; $F$ - final approval of the article
}

\section{Address for correspondence}

Ling Sun

E-mail: sunling6299@163.com

\section{Funding sources}

None declared

Conflict of interest

None declared

\section{Acknowledgements}

The authors are grateful to Dr. Abraham Morse for his English language editorial assistance.

Received on 0ctober 18, 2017

Reviewed on May 8, 2018

Accepted on May 14, 2018

Published online on January 31, 2019
Cite as

Wang $\mathrm{H}, \mathrm{Ou} \mathrm{Z}$, Chen Z, Yang L, Sun L. Influence of different post-thaw culture time on the clinical outcomes of different quality embryos. Adv Clin Exp Med. 2019;28(4):523-527. doi:10.17219/acem/91010

DOI

10.17219/acem/91010

\section{Copyright}

Copyright by Author(s)

This is an article distributed under the terms of the

Creative Commons Attribution Non-Commercial License

(http://creativecommons.org/licenses/by-nc-nd/4.0/)

\begin{abstract}
Background. In thawed embryo transfer cycles, the most common method is to transfer the embryos after $2 \mathrm{~h}$ of culture. Clinical outcomes of frozen-thawed cleavage embryo transfer cycles regarding the embryos status and the culture time of frozen-thawed cleavage embryos were limited and did not elucidate all unclear issues.
\end{abstract}

Objectives. The objective of this study was to examine the clinical outcomes of frozen-thawed cleavage embryo transfer cycles according to the embryos status and the culture time ( $2 \mathrm{~h}$ or overnight).

Material and methods. In this retrospective study (5-year period), 1,654 frozen-thawed embryos were analyzed. Firstly, frozen-thawed cleavage embryos were divided into 2 groups according to their status as follows: with at least 1 optimal embryo and without optimal embryos. Secondly, both of them were divided into 2 groups according to the culture time ( $2 \mathrm{~h}$ or overnight). Age of the female, infertility factors, clinical pregnancy, implantation rate, and live birth rate were compared.

Results. There were no statistically significant differences in the pregnancy rate, the implantation rate, live birth rate, the miscarriage rate, and the ectopic pregnancy rate in each group. However, the implantation rate increased after $2 \mathrm{~h}$ of incubation (41.1\%) compared to overnight incubation (36.0\%) in the group with at least 1 optimal day-3 embryo $(p<0.05)$. The cancellation rate in the suboptimal day-3 embryos group $(9.1 \%)$ was higher than in the group containing at least 1 optimal embryo (0.2\%) for the long (overnight) culture $(p<0.05)$.

Conclusions. The implantation rate can be improved in the optimal day-3 embryos transferred after $2 \mathrm{~h}$ of culture, but not for suboptimal day-3 embryos. Some unnecessary transfers can be avoided after overnight culture because of no further cleavage of the embryos.

Key words: assisted reproductive technology, embryo transfer, vitrification, frozen-thawed, clinical outcomes 


\section{Introduction}

The first human pregnancy from a frozen embryo was achieved using a slow freezing protocol in $1983 .{ }^{1}$ Since then, an increasing proportion of pregnancies are conceived after frozen embryo transfer (FET). ${ }^{2,3}$ Embryo cryopreservation can help reduce multiple birth rate following assisted human reproduction and can also maximize the effectiveness of the in vitro fertilization (IVF) cycles. ${ }^{4}$ Similarly, embryo cryopreservation is a crucial tool in cases of canceled embryo transfer (ET) due to ovarian hyperstimulation, endometrial bleeding, elevated serum progesterone levels on the day of triggering, preimplantation genetic screening (PGS), preimplantation genetic diagnosis (PGD), or any other unplanned events. ${ }^{5-7}$

In thawed embryo transfer cycles, the most common method is to transfer the embryos after $2 \mathrm{~h}$ of culture. One study suggested that a short post-thaw culture period is associated with higher implantation and live birth rates. ${ }^{8}$ However, compared to studies without further development during culture, several papers showed that significantly higher pregnancy and delivery rate was observed after transferring frozen-thawed embryos that had undergone cleavage requiring an overnight culture after thawing followed by transfer of the developing embryo. ${ }^{9,10}$ In addition, another study indicated that the pregnancy outcomes for embryos thawed and cultured overnight before transfer and those thawed and transferred on the same day are the same. ${ }^{11}$ These studies focused only on comparing the clinical outcomes following frozen-thawed transfer of embryos with different post-thaw culture time. However, we wondered whether the post-thaw culture time should vary according to the embryo quality before freezing.

In this study, we retrospectively compared the clinical outcomes of 2-hour culture with overnight culture in good quality vs lesser quality embryos to explore if the embryos with different qualities should be treated differently after thawing.

\section{Material and methods}

\section{Study design}

This study was declared exempt by the ethics committee of the Guangzhou Women and Children's Medical Center, China. Due to the retrospective nature of this study, patients' informed consent was not needed. A total of 1,654 cycles of in vitro fertilization (IVF) or intracytoplasmic sperm injection (ICSI) frozen-thawed transfer cycles (vitrified-freezing) performed at our hospital from May 2010 to May 2015 were retrospectively analyzed. The causes of infertility before embryo transfer included female factors, male factors, combined factors, and unexplained infertility.

Morphology of day- 3 embryos before vitrified-freezing were assessed according to the Istanbul consensus. ${ }^{12}$
Embryos with 7-8 equally sized mononucleated blastomeres and $<10 \%$ fragmentation were defined as optimal embryos. We divided the 1,654 cycles into 4 groups based on the quality of the embryos and the culture time before freezing. Group A1 ( $\mathrm{n}=321)$ had at least 1 optimal embryo on day 3 transferred after 2 h of thawing. Group A2 $(\mathrm{n}=531)$ had at least 1 optimal embryo on day 3 cultured overnight (about 24 h) before FET. Group B1 ( $\mathrm{n}=196)$ had no optimal day-3 embryos transferred after $2 \mathrm{~h}$ of thawing. Group B2 $(\mathrm{n}=541)$ had no optimal day 3 embryos cultured overnight before FET (Fig. 1).

\section{Cleavage stage embryo cryopreservation and thawing}

Vitrification was used for the cryopreservation of the embryos according to the manufacturer's protocol (ARSCI Inc., Longueuil, Canada). Embryo grading was done based on morphology: grade A - less than 10\% fragmentation and equal blastomeres; grade B - 10-30\% fragmentation and equal blastomeres; grade $\mathrm{C}-30-50 \%$ fragmentation and/or unequal blastomeres; and grade D - more than $50 \%$ fragmentation. ${ }^{13}$ Our strategy was to freeze the supernumerary embryos only if they exhibited a favorable grading with less than 30\% fragmentation of the blastomeres (grade A and B). Embryo quality was evaluated again after thawing and before transfer. The evaluation criteria for morphological survival of these frozen-thawed embryos were as follows: more than 50\% of the initial number of blastomeres intact and no signs of damage to the zona pellucida; otherwise thawing was considered to have failed. ${ }^{13}$ All of the surviving embryos were cultured in G1/G2-Plus medium (Vitorlife AB, Västra Frölunda, Sweden) after thawing; parts of the embryos were transferred after $2 \mathrm{~h}$ of thawing. For the embryos undergoing overnight culture, only embryos with further cleavage of at least 1 cell were considered viable for transfer.

\section{Endometrium preparation and thawed embryo transfer}

The endometrium was prepared for transfer in one of two ways: hormone replacement therapy (HRT) or natural cycle (NC). Patients with regular ovulation were treated with FET in an NC. A transvaginal ultrasound was used to monitor follicular growth and endometrial thickness on day 10 of the menstrual cycle. If the endometrial thickness reached $8 \mathrm{~mm}$ or more, the cycle was considered suitable for FET. Frozen embryo transfer was performed in an HRT cycle if patients did not ovulate regularly. Oral estrogen $6 \mathrm{mg} /$ day was commenced on the $3^{\text {rd }}$ day of the menstrual cycle for 12 days. When the endometrial thickness reached $8 \mathrm{~mm}$ or more, injections of progesterone at $60 \mathrm{mg} /$ day were administered for 3 days before FET, and the dosage was maintained after FET. ${ }^{14}$ 


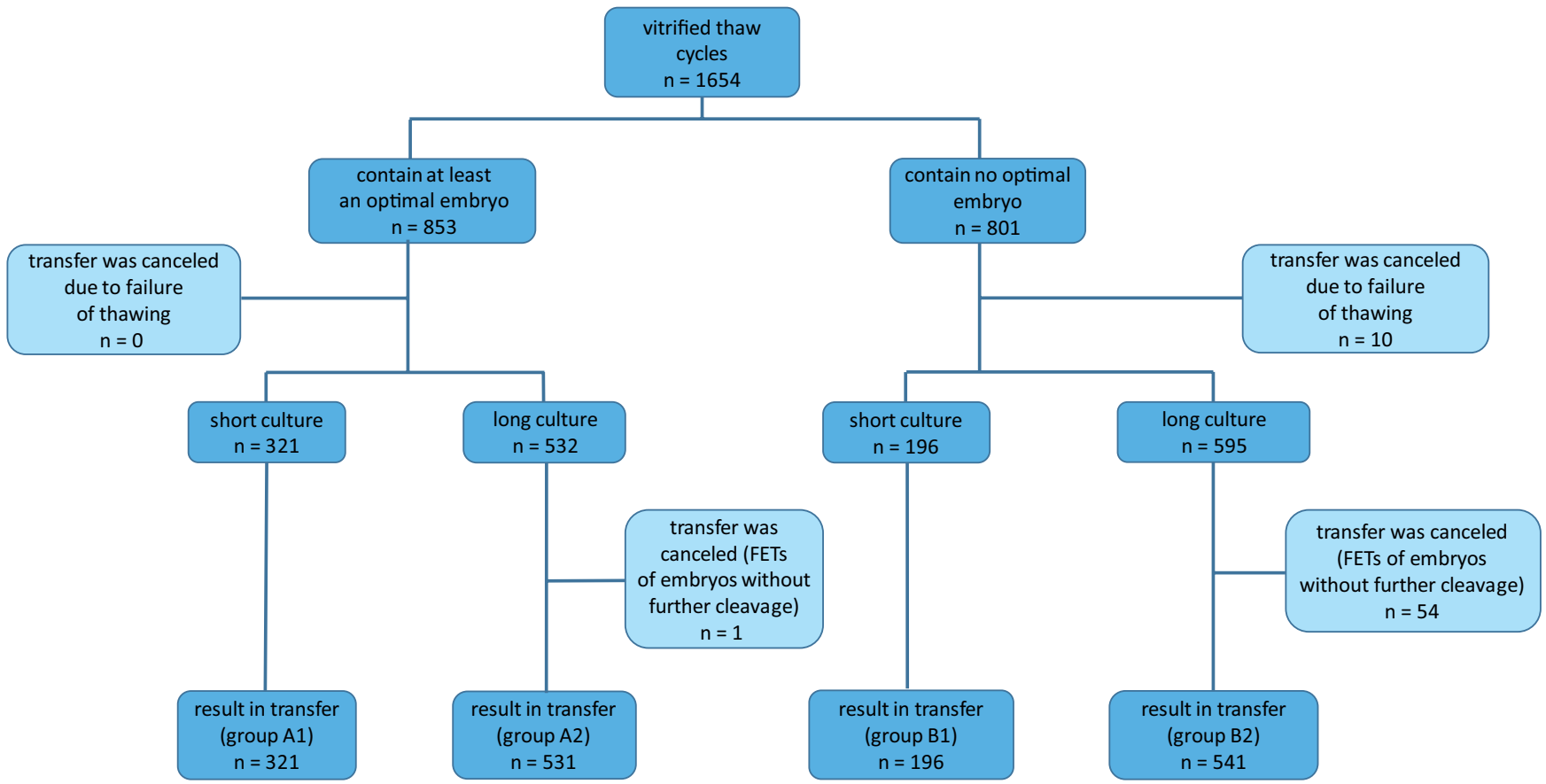

Fig. 1. Flow chart summarizing thawed embryo accountability of the study. The groups were divided by 2 aspects: the quality of the embryos and the culture duration after thawing

\section{Pregnancy outcome}

Clinical pregnancies (CP) were defined after observation of a gestational sac with or without a fetal heartbeat on ultrasound evaluation 4 weeks after FET. The number of sacs was taken as the number of successful implantations. Clinical miscarriage was defined when a pregnancy failed to progress after an intrauterine gestational sac had been detected with a pelvic ultrasonography. Live birth rate was defined as number of live births per number of FET. Ectopic pregnancy rate was also calculated.

\section{Statistical analysis}

Statistical analysis was performed using SPSS software v. 19 for Windows (SPSS Inc., Chicago, USA), applying parametric and nonparametric tests when appropriate. Continuous variables were expressed as mean \pm standard deviation (SD) and analyzed using Student's t-test. Categorical variables were expressed as percentages and analyzed using $X^{2}$ or Fisher's exact test depending on the sample size. Statistical significance was defined as p-values less than 0.05 .

\section{Results}

\section{Comparison of demographic characteristics}

There was no significant difference between the groups regarding age, body mass index (BMI) as well as basal follicle-stimulating hormone (FSH), and estradiol (E2) levels $(\mathrm{p}>0.05)$ (Table 1$)$.

\section{Analysis of immediate morphological survival and further cleavage}

Out of a total of 1,654 cycles, 853 cycles contained at least 1 optimal embryo. Eight hundred and one cycles had no optimal embryos. Three hundred and twenty-one of the thawed cycles were transferred after $2 \mathrm{~h}$ of culture (group A1). In group A2, 1 FET cycle was canceled because no further cleavage was observed, so the total number of transfers was 531. In groups B1 and B2, 10 cycles were canceled because of thawing failure. In group B1, 196 FET cycles were transferred after $2 \mathrm{~h}$ of culture; in group B2, 54 FET cycles were canceled, because no further cleavage was observed for a total of 541 embryo transfers. The cancellation rate for group B (9.1\%) was statistically significantly higher than in group A $(0.2 \%)(\mathrm{p}<0.05)$ (Table 2$)$.

\section{Clinical outcomes}

The clinical outcomes of 2 post-thaw cultures for optimal day-3 embryos (A1 vs A2) and suboptimal day-3 embryos (B1 vs B2) were compared. The pregnancy rate, live birth rate, miscarriage rate, and ectopic pregnancy rate were similar between the 2 groups but the implantation rate was higher after $2 \mathrm{~h}$ of incubation (41.1\%) compared to overnight incubation $(36.0 \%)(\mathrm{p}<0.05)$.

The pregnancy rate, live birth rate, miscarriage rate, ectopic pregnancy rate, and implantation rate of suboptimal 
Table 1. Comparison of demographic characteristics of 2 post-thaw culture lengths for different quality of day-3 embryos

\begin{tabular}{|c|c|c|c|c|c|c|}
\hline \multirow[b]{2}{*}{ Parameter } & \multicolumn{3}{|c|}{ At least 1 optimal embryo } & \multicolumn{3}{|c|}{ No optimal embryo } \\
\hline & $\begin{array}{l}\text { short culture } \\
\qquad(\mathrm{n}=321)\end{array}$ & $\begin{array}{l}\text { long culture } \\
\quad(n=531)\end{array}$ & $p$-value & $\begin{array}{l}\text { short culture } \\
\quad(n=196)\end{array}$ & $\begin{array}{l}\text { long culture } \\
\quad(n=541)\end{array}$ & $\mathrm{p}$-value \\
\hline EMT-BMI & $21.10 \pm 2.88$ & $20.89 \pm 2.68$ & NS (0.302) & $21.06 \pm 2.39$ & $20.82 \pm 2.62$ & NS $(0.277)$ \\
\hline Basic FSH & $5.66 \pm 3.04$ & $5.77 \pm 2.32$ & NS (0.559) & $5.85 \pm 2.01$ & $5.93 \pm 2.04$ & NS (0.639) \\
\hline
\end{tabular}

NS - not significant; EMT-BMI - body mass index; FSH - follicle stimulating hormone; Basic E2 - estradiol.

Table 2. Analysis of immediate morphological survival and further cleavage for different quality of day-3 embryos

\begin{tabular}{|l|c|c|}
\multicolumn{1}{|c|}{ Parameter } & $\begin{array}{c}\text { At least an optimal embryo } \\
(\mathrm{n}=853)\end{array}$ & $\begin{array}{c}\text { No optimal embryo } \\
(\mathrm{n}=801)\end{array}$ \\
\hline Failure of thawing rate [\%] & $0 / 853(0)$ & $10 / 801(0.01)$ \\
\hline $\begin{array}{l}\text { Cancel rate without further cleavage [\%] } \\
\text { (only for long culture) }\end{array}$ & $1 / 532(0.2)$ & 5.001 \\
\hline
\end{tabular}

day-3 embryos were similar between 2 different post-thaw culture groups $(\mathrm{p}>0.05)$ (Table 3$)$.

\section{Discussion}

All optimal embryos survived after thawing; however, 10 transfers were canceled due to failed thawing in the groups with only suboptimal embryos. We hypothesize that optimal embryos may better cope with stress during the frozen-thawed procedure, so high-quality embryos may suffer less damage during the process of cryopreservation and low-quality embryos may suffer more blastomere damage during the frozen-thawed procedure.

At present, the most common frozen-thawed transfer cycle method is to thaw the embryos and transfer them after $2 \mathrm{~h}$ of culture. The blastomere survival after thawing only can be identified in 2-hour culture. Furthermore, some studies reported that the developing potential of the thawed embryos appears to rely upon the resumption of mitosis, which may require a longer culture, generally overnight. ${ }^{15,16}$ Other studies also reported significantly better clinical outcomes after a longer culture time. ${ }^{17,18}$
Other reports found that cleavage-arrested embryos at $24 \mathrm{~h}$ following thawing were nevertheless still able to show some signs of further cleavage up to $48 \mathrm{~h}$ after thawing and could be implanted, but these embryos may have had a higher rate of chromosomal aberrations and more frequently ended in clinical abortion. ${ }^{19-21}$ In this study, there was only 1 canceled cycle in group A2 (at least 1 optimal embryo, overnight culture), but in group B2 (suboptimal embryos, overnight culture), 54 thawed cycles were canceled because no future cleavage was observed after overnight culture. This suggests that optimal embryos may have stronger development potential. If these 54 cycles had been transferred within $2 \mathrm{~h}$ of thawing, it may have resulted in an early pregnancy loss or fetal anomaly because of chromosomal aberrations and waste of resources. For the cycles without optimal embryos, it may be beneficial to prolong culture time (to overnight) after thawing in order to observe initial cleavage, so that some unnecessary transfers can be avoided.

We also compared the clinical outcomes of the 2-hour culture with the overnight culture to explore the specific effect of post-thaw culture time. We found that in the group with at least 1 optimal day-3 embryo, there

Table 3. Comparison of the clinical outcomes of 2 post-thaw culture times for different quality of day-3 embryos

\begin{tabular}{|c|c|c|c|c|c|c|}
\hline \multirow[b]{2}{*}{ Parameter } & \multicolumn{3}{|c|}{ At least 1 optimal embryo } & \multicolumn{3}{|c|}{ No optimal embryo } \\
\hline & $\begin{array}{l}\text { short culture } \\
\quad(n=321)\end{array}$ & $\begin{array}{l}\text { long culture } \\
\qquad(n=531)\end{array}$ & $\mathrm{p}$-value & $\begin{array}{l}\text { short culture } \\
\quad(n=196)\end{array}$ & $\begin{array}{l}\text { long culture } \\
\qquad(\mathrm{n}=541)\end{array}$ & $\mathrm{p}$-value \\
\hline Clinical pregnancy rate [\%] & $189 / 321(58.9)$ & 285/531 (53.7) & NS (0.14) & $68 / 196(34.7)$ & 206/541 (38.1) & NS (0.40) \\
\hline Implantation rate [\%] & $280 / 682(41.1)$ & $391 / 1087(36.0)$ & 0.03 & $89 / 414(21.5)$ & $262 / 1115(23.5)$ & NS (0.41) \\
\hline Miscarriage rate [\%] & 23/189 (12.2) & $30 / 285(10.5)$ & NS (0.58) & $14 / 68(20.6)$ & $34 / 206(16.5)$ & NS (0.44) \\
\hline Ectopic pregnancy rate [\%] & $11 / 189(5.8)$ & $18 / 285(6.3)$ & NS $(0.83)$ & $5 / 68(7.4)$ & $11 / 206(5.3)$ & NS (0.54) \\
\hline Live birth rate [\%] & $155 / 321(48.3)$ & $237 / 531(44.6)$ & NS (0.29) & 49/196 (25.0) & $161 / 541(29.8)$ & NS $(0.20)$ \\
\hline
\end{tabular}

NS - not significant. 
was no significant influence of culture time on most clinical outcomes, but the implantation rate increased after $2 \mathrm{~h}$ of incubation compared with that of the overnight incubation group, which was consistent with the findings from a previous study. ${ }^{8}$ The result of the increasing implantation rate after $2 \mathrm{~h}$ of incubation may be associated with the environmental impact on the potential of embryo development. Although embryo culture aims to mimic the fallopian tube and uterine environment, it always implies the induction of some related stress. ${ }^{13,22,23}$ It thus seems reasonable to hypothesize that frozen-thawed suboptimal embryos may be less able to adapt to suboptimal environments such as the currently available culture systems. We also hypothesize that the suboptimal embryos may express oxidative stress in the cryopreservation medium, and higher oxidative stress levels are associated with lower implantation rates. ${ }^{24}$ Therefore, we think that the implantation rate for optimal day- 3 embryo transfers after $2 \mathrm{~h}$ of culture can be improved.

\section{Conclusions}

The conclusions from this study are based on retrospective data and have the inherent limitations of retrospective studies. For optimal quality embryos, $2 \mathrm{~h}$ of post-thaw culture appear to optimize clinical outcomes; however, for embryos of suboptimal quality, some unnecessary transfer of non-functional day-3 embryos can be avoided after overnight culture which allows for the identification of embryos that fail to undergo additional cleavage.

\section{References}

1. Trounson A, Mohr L. Human pregnancy following cryopreservation, thawing and transfer of an eight-cell embryo. Nature. 1983;305(5936): 707-709.

2. Ferraretti AP, Goossens V, de Mouzon J, et al; European IVF-monitoring (EIM); Consortium for European Society of Human Reproduction and Embryology (ESHRE). Assisted reproductive technology in Europe, 2008: Results generated from European registers by ESHRE. Hum Reprod. 2012;27(9):2571-2584.

3. Sullivan EA, Zegers-Hochschild F, Mansour R, et al. International Committee for Monitoring Assisted Reproductive Technologies (ICMART) world report: Assisted reproductive technology 2004. Hum Reprod. 2013;28(5):1375-1390.

4. Tiitinen A. Prevention of multiple pregnancies in infertility treatment. Best practice \& research. Best Pract Res Clin Obstet Gynaecol. 2012;26(6):829-840.

5. Amso NN, Ahuja KK, Morris N, Shaw RW. The management of predicted ovarian hyperstimulation involving gonadotropin-releasing hormone analog with elective cryopreservation of all pre-embryos. Fertil Steril. 1990;53(6):1087-1090.
6. Wong KM, Mastenbroek S, Repping S. Cryopreservation of human embryos and its contribution to in vitro fertilization success rates. Fertil Steril. 2014;102(1):19-26.

7. Yang S, Pang T, Li R, et al. The individualized choice of embryo transfer timing for patients with elevated serum progesterone level on the HCG day in IVF/ICSI cycles: A prospective randomized clinical study. Gynecol Endocrinol. 2015;31(5):355-358.

8. Rato ML, Gouveia-Oliveira A, Plancha CE. Influence of post-thaw culture on the developmental potential of human frozen embryos. J Assist Reprod Genet. 2012;29(8):789-795.

9. Joshi BV, Banker MR, Patel PM, Shah PB. Transfer of human frozenthawed embryos with further cleavage during culture increases pregnancy rates. J Hum Reprod Sci. 2010;3(2):76-79.

10. Guerif F, Cadoret V, Poindron J, Lansac J, Royere D. Overnight incubation improves selection of frozen-thawed blastocysts for transfer: Preliminary study using supernumerary embryos. Theriogenology. 2003;60(8):1457-1466.

11. Fang C, Yue CM, Huang R, Wei LN, Jia L. Pregnancy outcomes of blastocysts cultured overnight after thawing. Arch Obstet Gynecol. 2016; 293(6):1347-1356.

12. ALPHA Scientists In Reproductive Medicine; ESHRE Special Interest Group Embryology. Istanbul consensus workshop on embryo assessment: Proceedings of an expert meeting. Reprod Biomed Online. 2011; 22(6):632-646.

13. Chian R-C. Fertility Cryopreservation. Cambridge, UK: Cambridge University Press; 2010:89-95.

14. Groenewoud ER, Cantineau AE, Kollen BJ, Macklon NS, Cohlen BJ. What is the optimal means of preparing the endometrium in frozenthawed embryo transfer cycles? A systematic review and meta-analysis. Hum Reprod Update. 2013;19(5):458-470.

15. Ziebe S, Bech B, Petersen K, Mikkelsen AL, Gabrielsen A, Andersen AN. Resumption of mitosis during post-thaw culture: A key parameter in selecting the right embryos for transfer. Hum Reprod. 1998;13(1): 178-181.

16. Guerif F, Bidault R, Cadoret V, Couet ML, Lansac J, Royere D. Parameters guiding selection of best embryos for transfer after cryopreservation: A reappraisal. Hum Reprod. 2002;17(5):1321-1326.

17. Sifer C, Sellami A, Poncelet C, et al. Day 3 compared with day 2 cryopreservation does not affect embryo survival but improves the outcome of frozen-thawed embryo transfers. Fertil Steril. 2006;86(5): 1537-1540.

18. Hartshorne GM, Elder K, Crow J, Dyson H, Edwards RG. The influence of in-vitro development upon post-thaw survival and implantation of cryopreserved human blastocysts. Hum Reprod. 1991;6(11):136-141.

19. Van der Elst J, Van den Abbeel E, Vitrier S, Camus M, Devroey P, Van Steirteghem AC. Selective transfer of cryopreserved human embryos with further cleavage after thawing increases delivery and implantation rates. Hum Reprod. 1997;12(7):1513-1521.

20. Laverge H, Van der Elst J, De Sutter P, Verschraegen-Spae MR, De Paepe A, Dhont M. Fluorescent in-situ hybridization on human embryos showing cleavage arrest after freezing and thawing. Hum Reprod. 1998;13(2):425-429.

21. Munne S, Alikani M, Tomkin G, Grifo J, Cohen J. Embryo morphology, developmental rates, and maternal age are correlated with chromosome abnormalities. Fertil Steril. 1995;64(2):382-391.

22. Leese HJ, Sturmey RG, Baumann CG, McEvoy TG. Embryo viability and metabolism: Obeying the quiet rules. Hum Reprod. 2007;22(12): 3047-3050.

23. Leese HJ, Baumann CG, Brison DR, McEvoy TG, Sturmey RG. Metabolism of the viable mammalian embryo: Quietness revisited. Mol Hum Reprod. 2008;14(12):667-672.

24. Wiener-Megnazi Z, Lahav-Baratz S, Blais I, et al. Oxidative markers in cryopreservation medium from frozen-thawed embryos: A possible tool for improved embryo selection in in vitro fertilization? J Assist Reprod Genet. 2016;33(6):731-739. 